\title{
Socialisation sexuelle des jeunes issus des migrations subsahariennes en Suisse
}

Transformations générationnelles des sexualités

Michela Villani, Francesca Poglia Mileti, Laura Mellini et Brikela Sulstarova

\section{OpenEdition}

\section{Journals}

Édition électronique

URL : http://journals.openedition.org/anthropodev/791

DOI : 10.4000/anthropodev.791

ISSN : 2553-1719

Éditeur

Presses universitaires de Louvain

Édition imprimée

Date de publication : 1 décembre 2019

Pagination : 11-29

ISBN : 978-2-87558-940-8

ISSN : 2276-2019

\section{Référence électronique}

Michela Villani, Francesca Poglia Mileti, Laura Mellini et Brikela Sulstarova, « Socialisation sexuelle des jeunes issus des migrations subsahariennes en Suisse », Anthropologie \& développement [En ligne], 50 । 2019, mis en ligne le 19 décembre 2020, consulté le 24 janvier 2021. URL : http:// journals.openedition.org/anthropodev/791; DOI : https://doi.org/10.4000/anthropodev.791

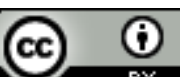

La revue Anthropologie \& développement est mise à disposition selon les termes de la Licence Creative Commons Attribution 4.0 International. 


\title{
Socialisation sexuelle des jeunes issus des migrations subsahariennes en Suisse
}

\author{
Transformations générationnelles des sexualités
}

\author{
Michela Villani ${ }^{1}$, Francesca Poglia Mileti ${ }^{2}$, Laura Mellini ${ }^{3}$ \\ Brikela Sulstarova $^{4}$
}

\begin{abstract}
Comme d'autres pays européens, la Suisse est confrontée à des changements liés aux flux migratoires concernant tout particulièrement la jeunesse. Cet article s'intéresse aux jeunes issu.es des migrations subsahariennes (première et deuxième génération) vivant en Suisse. Basé sur une recherche qualitative, cet article porte sur la socialisation sexuelle, à savoir les dynamiques d'entrée dans la sexualité, et met en lumière les comportements sexuels ainsi que la gestion des différences culturelles et les tensions intergénérationnelles en contexte de migration. À partir des expériences subjectives des jeunes interviewées, l'analyse montre comment ces dernier.es se positionnent face aux normes sexuelles transmises par les parents et à celles circulant au sein des sociabilités juvéniles, où l'activité sexuelle a lieu. Trois points seront illustrés : la socialisation familiale et le rôle des parents comme agents de socialisation; la socialisation entre pairs au travers d'une perspective de genre; la gestion des contradictions entre les normes transmises par les parents et celles circulant dans les groupes de pairs.
\end{abstract}

As other European countries, Switzerland is facing changes related to migration flows, especially concerning youth. This article focuses on first and second generation young immigrants from sub-Saharan Africa living in Switzerland. Based on qualitative research, this article brings on sexual socialization, namely how young immigrants enter into sexuality, and highlights how they deal with cultural differences and intergenerational tensions related to their sexual behaviors in the migratory context. Based on the subjective experiences of young interviewees, the analysis shows how they position themselves against or in favor of sexual norms transmitted by parents and those circulating within peer groups. Three points will be illustrated: the family socialization and the role of parents as agents of socialization in relation to sexuality; the peer socialization through a gender perspective; the management of contradictions between sexual norms transmitted by parents and those circulating among the peer groups.

\footnotetext{
1 Chercheuse senior, département des sciences sociales, université de Fribourg, Suisse ; michela.villani@unifr.ch

2 Professeure de sociologie, département des sciences sociales, université de Fribourg, Suisse ; francesca.poglia@unifr.ch

3 Chercheuse senior, département des sciences sociales, université de Fribourg, Suisse : laura.mellini@unifr.ch

${ }^{4}$ Chercheuse senior, université de Fribourg, Suisse ; brikela.sulstarova@unifr.ch
} 


\section{Introduction}

À l'instar d'autres pays européens, on observe en Suisse une forte augmentation des ressortissant·es provenant des pays extra-européens, leur proportion ayant doublé ces vingt dernières années. Cette augmentation touche également les populations issues d'Afrique subsaharienne, au centre de cet article. À titre indicatif, en 2017, on recensait en Suisse 107051 personnes nées dans un pays d'Afrique subsaharienne, dont la part de jeunes âgé-es entre 18 et 24 ans représentait $14 \%$ (15 031), répartis entre une majorité $d^{\prime}$ hommes $(9070)$ et une minorité de femmes $(5961)^{5}$. Si les populations originaires d'Afrique subsaharienne qui migrent en Suisse présentent des parcours migratoires et des caractéristiques sociodémographiques très hétérogènes (Bolzman et al., 2011 ; EfionayiMäder et al., 2005), les médias s'intéressent particulièrement à la situation des requérant.es d'asile subsaharien-nes. En se réappropriant des statistiques récentes (Efionayi-Mäder et al., 2011), les médias dressent souvent des portraits de jeunes hommes en situation de précarité et annoncent des nouveaux dangers pour l'ordre public ou des risques en termes de santé publique. Finalement la race, sans être jamais mentionnée, apparaît en filigrane dans les manières de singulariser, voire de discriminer les populations subsahariennes dans les politiques sociales (Efionayi-Mäder et Ruedin, 2017).

Désignés comme une population vulnérable, ces jeunes deviennent la cible des études épidémiologiques dont les enjeux sont décrits comme importants (Wandeler et al., 2008), et leur sexualité est appréhendée purement en termes de comportements à risque. Aucune recherche qualitative ne s'est intéressée jusqu'ici à leur socialisation sexuelle, à savoir la manière dont les jeunes en Suisse entrent dans la sexualité. Si les questions liées aux comportements sexuels et à la prévention des maladies sexuellement transmissibles (MST) sont explorées par des recherches d'empreinte médicale (Barrense-Dias et al., 2018), les aspects entourant la communication autour de la sexualité, ainsi que la transmission des normes sexuelles, ne sont que peu étudiés par les scientifiques dans ce contexte particulier (Colombo et al., 2017). Notre enquête met en lumière les processus de socialisation sexuelle, les comportements sexuels et les représentations des jeunes, ainsi que la manière de gérer les différences culturelles et les tensions intergénérationnelles en contexte de migration. S'intéresser à la socialisation sexuelle des jeunes subsaharien.nes vivant en Suisse permet aussi de montrer comment l'origine qualifie et reproduit les inégalités dans les trajectoires sexuelles (Desgrées du Loû et Lert, 2017).

En sciences sociales, la sexualité est étudiée comme une activité sociale, dont l'importance ne tient pas qu'aux actes (les comportements) mais tient aussi aux significations attribuées à ces derniers (Bozon, 1999). La sexualité, loin d'être considérée

\footnotetext{
${ }^{5}$ Données produites à partir de la base de données STAT-POP de I'Office fédéral de la statistique, disponible sur le site https://www.bfs.admin.ch/bfs/fr/home/services/recherche/stat-tab-donneesinteractives.html (mis à jour le 3 septembre 2018).
} 
comme l'issue d'une pulsion sexuelle, un besoin ou un instinct, est ainsi appréhendée comme le résultat d'un processus de socialisation : de l'acquisition d'un savoir-faire sexuel (apprendre des pratiques) à la formation du goût, voire du désir sexuel lié à l'intériorisation des normes morales. En effet, la socialisation sexuelle induit un façonnement de la personnalité de l'individu, tout comme de sa sensibilité et de ses goûts (Elias, 1973), l'apprentissage ne concernant pas uniquement des techniques corporelles se traduisant en pratiques sexuelles - mais concernant aussi une morale, l'acquisition de normes (Marquet, 2004), l'intériorisation du licite et de l'illicite. Durant leur socialisation sexuelle, les jeunes façonnent donc leur subjectivité à partir des normes, valeurs, symboles et du langage offerts par les agents de socialisation (Bozon, 2013 ; Giddens, 1992 ; Weeks, 2014). Dès lors, analyser le processus de socialisation signifie rendre compte du rôle des agents impliqués dans le processus, lors duquel les jeunes acquièrent des notions, mais développent également des représentations imprégnées de la culture et des valeurs morales et religieuses transmises par ces mêmes agents de socialisation.

Face à des codes de comportements multiples et des normes sexuelles parfois contradictoires, les jeunes sont amené-es à gérer des tensions intergénérationnelles, des conflits de loyauté et des comportements risqués en matière de santé sexuelle. Basé sur les résultats d'une recherche qualitative, cet article se fonde sur un corpus d'une cinquantaine d'entretiens approfondis menés avec des hommes et des femmes, âgé.es entre 18 et 25 ans, de première et de deuxième génération issu-es de la migration subsaharienne ${ }^{6}$. Le texte aborde ces thématiques par le biais de la socialisation sexuelle et centre l'analyse sur les deux agents principaux de la socialisation sexuelle : les parents et les pairs. À partir des expériences subjectives des jeunes interviewé·es, l'analyse montre comment ces dernier-es se positionnent face aux normes sexuelles transmises par les parents et celles circulant au sein des sociabilités juvéniles, où l'activité sexuelle a lieu. En particulier, trois points seront illustrés.

Le premier aborde la socialisation familiale et le rôle des parents comme agents de socialisation. Il montre que la socialisation à la parole sexuelle - ou le fait de parler de sexualité - est le résultat d'un apprentissage. Dans ce cadre, la comparaison entre première et deuxième génération met en lumière comment se construisent les références en matière de sexualité lorsque les contextes de socialisation familiale diffèrent. Le deuxième point se focalise sur la socialisation entre pairs et adopte une perspective de genre pour montrer que, en dépit de rôles et discussions genrés, il y a une évolution des rapports de genre déclenchée par l'expérience migratoire. II indique aussi qu'il y a des différences entre première et deuxième génération dans la manière de gérer certains sujets de discussions (contraception, masturbation) entre pairs. Le troisième point

\footnotetext{
${ }^{6}$ Nous considérons comme deuxième génération les enfants de migrant·es né·es à l'étranger (dans un pays d'Afrique subsaharienne) ainsi que celles et ceux qui sont arrivé·es en Suisse avant l'âge de 10 ans et qui ont été scolarisé.es en Suisse. Ce choix se justifie par le fait que la socialisation sexuelle intervient à partir de cet âge, y compris dans le cadre scolaire, ayant donc une incidence sur les activités et les représentations sexuelles. Dans notre enquête, 24 jeunes sont de première génération (dont 11 femmes et 13 hommes) et 23 jeunes de deuxième génération (dont 15 femmes et 8 hommes).
} 
concerne la gestion des normes contradictoires transmises par les parents ou circulant entre les groupes de pairs. Nous verrons que, face aux nombreuses injonctions faites aux jeunes femmes en particulier (de la part des parents, des pairs, des professionnels, des médias), l'origine subsaharienne est évoquée pour réinscrire les relations sexuelles dans des représentations inégalitaires et altérisées.

\section{Enquêter la sexualité en Suisse : choix et enjeux méthodologiques}

L'enquête JASS (Jeunes originaires d'Afrique subsaharienne et santé sexuelle) ${ }^{7}$ a eu pour objectif d'explorer les contextes et les agents de socialisation sexuelle en lien avec les choix et comportements sexuels (prévention du $\mathrm{VIH}$, pratiques à risque, etc.) des jeunes issu.es de la migration subsaharienne. Caractérisée par la variété de langues et les différences cantonales, la Suisse n'a pas une tradition uniforme de recherche sur la sexualité sur l'ensemble de son territoire. Les enquêtes conduites sur les comportements sexuels sont souvent menées en fonction de la région géographique, ancrées dans des traditions francophones ou germanophones. Les études sont fragmentées et souvent justifiées par des problématiques associées à la recherche médicale, qui met en avant la notion de risque, qu'il soit de nature sanitaire (Campisi et al., 2017) ou sociale (Colombo et al., 2017 ; Poglia Mileti et al., 2019). Si l'enquête a initialement été justifiée par des arguments épidémiologiques (Wandeler et al., 2008), très rapidement nous nous sommes rendu compte de l'importance d'explorer d'autres éléments, moins visibles et pourtant très présents dans le vécu des jeunes : le sentiment d'être différent.es. Différent.es de qui ? De la population majoritaire, de ceux que les jeunes indiquent par l'appellation « les $100 \%$ suisses $"$. II nous a donc semblé d'autant plus important de nous concentrer sur les aspects liés à la socialisation, sur leurs connaissances de la sexualité mais aussi sur les normes transmises durant leur enfance, leur puberté et leur jeunesse.

Forte d'une expérience scientifique précédente (FEMIS) ${ }^{8}$, l'équipe JASS est constituée par un chercheur et quatre chercheuses, dont la formation réunit les disciplines de la sociologie, de la linguistique et du travail social. Adoptant une approche interdisciplinaire, les préoccupations des chercheuses n'étaient pas les mêmes, chacune ayant apporté une réflexion issue de sa discipline. Dès lors, il a été important de comprendre les dynamiques des inégalités et des facteurs y contribuant, les codes de la communication et le choix des mots, les retombées des résultats d'enquête pour les professionnel-les et notamment pour les travailleurs et travailleuses sociaux.

Durant toute l'enquête, une démarche réflexive a été adoptée, dans la ligne des travaux de terrain menés précédemment par les enquêtrices sur des sujets intimes (sexualité,

\footnotetext{
${ }^{7}$ Recherche financée par le Fonds national suisse de la recherche scientifique (100017_162382) de 2016 à 2018, menée à l'université de Fribourg par l'équipe de recherche suivante : Francesca Poglia Mileti, Pascal Singy, Laura Mellini, Michela Villani, Brikela Sulstarova.

${ }^{8}$ II s'agit de l'enquête FEMIS, « Femmes migrantes d'origine subsaharienne et VIH : gestion d'un secret et rapport à la santé », financée par le Fonds national suisse et conduite entre 2012 et 2014.
} 
maladies, secrets, etc.) auprès de populations en situation de vulnérabilité (migrant·es, personnes séropositives, femmes excisées, hommes homosexuels, jeunes endettés). Comme pour l'enquête FEMIS, une attention particulière a été portée aux enjeux éthiques et méthodologiques liés au fait d'enquêter sur des sujets sensibles auprès de ces populations (Villani et al., 2014, 2015).

Ainsi, pour préparer l'entrée dans le terrain, l'équipe de recherche a organisé un focus group avec cinq expert.es travaillant dans les domaines de la santé sexuelle et de la migration et dans le milieu d'associations africaines : les questions discutées ont porté sur les lieux et les stratégies de recrutement, les approches à utiliser pour entrer en contact avec la population d'enquête, la relation d'enquête (notamment les différences d'âge, de sexe et d'origine entre enquêtrices et enquêté-es) et le guide d'entretien (thèmes abordés, question d'entrée en matière, formulation des questions, etc.). Si l'engagement d'enquêteurs (hommes) issus de la migration africaine avait été pris en considération par l'équipe de recherche, les expert.es ont souligné les avantages d'une équipe féminine et d'origine occidentale. En effet, avec les jeunes femmes, les enquêtrices peuvent jouer sur le registre de la complicité féminine, tandis que, pour les jeunes hommes, l'avantage d'une enquêtrice tient au fait qu'elle n'entre pas dans les logiques de compétition typiques des sociabilités masculines (Amsellem-Mainguy et Vuattoux, 2018). Quant à l'éventuelle proximité d'origine entre enquêtrices et enquêté.es, elle aurait risqué d'engendrer un effet d'autocensure chez les premières (gêne de poser certaines questions sachant, a priori, ce qui peut déranger) et de rester dans le registre de l'implicite chez les secondes (pas d'explicitation puisque l'interlocutrice est censée savoir de quoi on parle).

À partir de là, tous ces éléments de proximité/distance entre enquêtrices et enquêté.es ont fait l'objet de réflexions et d'analyses régulières au sein de l'équipe de recherche. Par ailleurs, à l'issue de chaque entretien, chacune des enquêtrices s'est engagée à produire deux ou trois pages de notes personnelles, plaçant au centre une analyse méthodologique de la situation d'entretien et de la relation d'enquête (Villani et al., 2014). Ces fiches de notes personnelles étaient ensuite lues par chacune des enquêtrices et faisaient l'objet de discussions collectives, afin d'analyser l'influence de l'écart entre enquêtrices et enquêté es (en termes d'âge, de sexe et d'origine) sur la production des données (Amsellem-Mainguy et Vuattoux, 2018).

Les entretiens se sont déroulés dans un contexte respectueux de la confidentialité et ont duré plusieurs heures, nécessitant parfois pour les interviewé.es de revenir pour la deuxième partie. L'anonymat a été garanti, les noms utilisés dans cet article sont fictifs. Au total, 47 entretiens approfondis ont été réalisé et deux focus groups (chacun avec cinq participant-es et séparant les jeunes de première génération de ceux de deuxième génération). Pour les personnes interviewées, une fiche sociodémographique a été remplie et nous avons analysé les variables telles que le niveau d'étude et le statut socioprofessionnel (des enquêté es et de leurs parents) ou la religion. La population dans son ensemble était équilibrée en termes de génération ( 24 individus de première génération et 23 de deuxième) et de genre ( 21 hommes et 26 femmes). Les pays d'origine étaient très variés pour un total de quinze pays concernés. Parmi les pays d'origine, 
27 jeunes provenaient de pays francophones et 20 de pays anglophones. Finalement la moitié de ces jeunes avaient un permis de résidence à vie voire la nationalité suisse (26 jeunes), une partie d'entre eux avaient un permis de séjour de longue durée (12 jeunes) et les restants ( 9 jeunes) se trouvaient dans une situation de forte précarité (permis de séjour de courte durée, attente de régularisation, sans permis). Sans surprise, les jeunes de deuxième génération ont un niveau d'étude plus élevé (secondaire, universitaire) que ceux de première. Toutefois parmi ces derniers, plusieurs jeunes ont repris les études ou une formation en Suisse. Concernant la religion, les appartenances sont variées, avec une prévalence de jeunes catholiques et de protestant·es (tableau 1). Quant à l'orientation sexuelle, seule une femme s'est dite "bisexuelle ", alors que l'ensemble de la population s'est déclarée de manière homogène " hétérosexuel·le ". Les efforts répétés des enquêtrices pour réunir un échantillon plus diversifié en termes d'orientation sexuelle n'ont pas donné les résultats escomptés, ce qui constitue sans doute une limite de cette étude.

Tableau 1 : Genre, génération et religion

\begin{tabular}{|c|c|c|c|c|c|c|}
\hline Religion & $\mathrm{F} \mathrm{G1}$ & $\mathrm{H} \mathrm{G1}$ & $\mathrm{F} \mathrm{G} 2$ & $\mathrm{H} \mathrm{G} 2$ & Total F (G1+G2) & Total H (G1+G2) \\
\hline Catholique & 2 & 2 & 8 & 6 & 10 & 8 \\
\hline Protestante & 3 & 1 & 4 & - & 7 & 1 \\
\hline Évangélique & 1 & 1 & 1 & - & 2 & 1 \\
\hline Chrétienne orthodoxe & 2 & 4 & 1 & - & 3 & 4 \\
\hline Musulmane & 2 & 5 & - & 2 & 2 & 7 \\
\hline Non-croyant·e & 1 & - & 1 & - & 2 & - \\
\hline
\end{tabular}

$F=$ femme $; H=$ homme $; G 1$ = première génération ; $G 2$ = deuxième génération.

\section{Les parents comme des agents de socialisation : parole sexuelle ou tabou}

Parler de sexualité ne va pas de soi : la capacité à produire une parole sexuelle est le résultat d'un apprentissage, le fruit d'une socialisation et est considéré comme un trait spécifique de la culture occidentale. On apprend à parler parce que des mots sont dits sur la sexualité, et les manières de construire ces récits varient selon les milieux mais aussi les contextes. Les mots peuvent être techniques ou exprimer des interdits, devenir moralisants ou inquiétants. À l'inverse, l'absence de mots ou le silence sont qualifiés par les jeunes de "tabous", ce qui est souvent signalé lorsqu'ils se réfèrent à la communication au sujet de la sexualité avec les parents (Sulstarova et al., 2019). Cette première partie explore le contexte socioculturel au sein duquel la parole sexuelle s'exprime, se nie ou évolue au cours du temps. Elle a pour objectif d'analyser les formes narratives qui relatent aussi bien des expériences que des savoirs autour du sexe, permettant ainsi d'analyser les stratégies communicationnelles selon leurs contextes d'émergence et d'utilisation. Le premier constat est que les messages parentaux sont souvent en décalage avec ceux entendus lors des leçons d'éducation sexuelle en milieu scolaire, ou circulant parmi les pairs (jeunes du même âge). 


\section{Entre paroles évitées et construction de la sexualité comme danger}

Les jeunes de première génération mettent en avant le fait que la question de la sexualité est difficile à aborder en famille. Des expressions telles que " la sexualité en Afrique est taboue » ou " on n'en parle pas » sont souvent mentionnées. En explorant les dynamiques de la communication sur ce sujet, toutefois, des codes se précisent selon l'interlocuteur ou l'interlocutrice. Parler de sexualité n'est pas négatif en soi : cela dépend avec qui on en parle. Par exemple, dans le contexte familial ou avec une personne plus âgée, parler de sexualité peut être perçu comme un manque de respect, une perte de contrôle de soi ou une forme d'addiction à la sexualité (être obsédé), comme l'explique Sam, un jeune Érythréen de 25 ans, en Suisse depuis un an, de religion chrétienne orthodoxe et en attente d'un permis stable :

Chez nous si on parle de sexualité, les gens vont penser que je suis une personne... addict par la sexualité... que je vais penser à ça tout le temps. Chez nous, dans les villages c'est interdit de parler comme ça, il y a aussi la timidité pour parler de ça. On est nouveaux ici, ça veut dire qu'on a commencé une nouvelle vie ici. C'est très différent : on parle pas de sexualité dans nos familles. C'est interdit de parler de choses comme ça, parce que c'est un manque de respect. (Sam)

Un autre élément important concerne la socialisation à la santé sexuelle, c'est-à-dire l'acquisition d'un savoir normatif et langagier issu du monde médical pour décrire les relations sexuelles. Selon le contexte sociopolitique et national dans lequel les jeunes ont grandi avant de migrer, les cours d'éducation sexuelle ont été dispensés par des organisations non gouvernementales (ONG) occidentales ou locales opérant en Afrique ou par les gouvernements locaux. Les lieux d'origine des enquêté.es sont multiples et variés, certain.es venant de contextes instables ou de zones en conflit. Les jeunes disent que ces campagnes de prévention et les messages transmis dans ces contextes sont quasi exclusivement axés sur la prévention à la violence basée sur le genre (VBG, telle que mutilations sexuelles, repassage des seins, viols, violences conjugales, etc.) ou sur le thème du VIH/sida. La sexualité est ainsi traitée en termes de risque potentiel, présentée comme une situation dangereuse.

En Afrique ils nous parlaient du viol, comment faire après être violée, on avait des informations par rapport à ça. Ils nous disaient qu'après il faut en parler, il faut aller à l'hôpital, il faut faire des examens et tout. Parce que ça arrivait beaucoup. C'était très très fréquent. On n'osait pas forcément aller en parler aux parents, parce qu'on avait peur de leur réaction. Peur qu'ils disent que c'est de notre faute, que nous l'avons cherché. (Kayla)

Kayla, une Camerounaise de 19 ans, arrivée en Suisse à l'âge de 12 ans, est catholique pratiquante, possède un permis de séjour stable, elle suit une formation professionnelle et vit avec sa mère. Elle met en exergue deux aspects essentiels de la socialisation sexuelle dans le contexte pré-migratoire: d'une part, la sexualité est abordée sous l'angle du danger, d'autre part, la peur d'en parler et de se voir attribuer par les parents une prétendue responsabilité : "C'est de notre faute, [que] nous l'avons cherché ". Si la violence faite aux femmes s'appuie sur ces mécanismes de la honte et de la culpabilité, ici 
ce mécanisme est renforcé par les cours "mettant en garde » les femmes contre les risques de viol.

Les jeunes de première génération disent adapter leurs pratiques au nouveau contexte de migration une fois arrivé.es en Suisse. Toutefois la difficulté à parler de sexualité (et parfois le manque de ressources linguistiques pour le faire) les pénalise par rapport aux autres jeunes socialisées dans ce contexte, ayant un langage technique et médical (Gardey et Vuille, 2018). Cette capacité spécifique (la valorisation occidentale de la parole et la construction de la sexualité en termes médicaux) devient un facteur défavorisant autant pour les hommes que pour les femmes migrant·es, notamment lorsqu'il s'agit de solliciter un service de santé (prise d'un contraceptif, accompagnement du ou de la partenaire, négociation du moyen de contraception, etc.). Les jeunes de deuxième génération, quant à eux, vantent une grammaire sexuelle plus étendue et diversifiée. De plus, ces messages sont discutés en famille et confirmés par les parents, également socialisés en Suisse, selon leur parcours de migration. Comme dans d'autres études menées sur les enfants d'immigrés en France ${ }^{9}$, les jeunes de notre enquête ressentent très souvent un décalage entre les messages reçus dans les enseignements d'éducation sexuelle (sexualité liée aux risques de grossesse, de MST, etc.), ceux qui sont transmis par les parents (injonctions à la virginité des filles, respect des codes religieux, pression à I'homogamie ethnique, etc.) et ceux qui sont discutés entre pairs.

Le manque de récit sur la sexualité au sein des familles ou sa pauvreté sont interprétés en termes de " tabou », ce qui est parfois confirmé par un langage moralisant venant de la religion et justifiant les interdits. À ce propos, nous avons relevé que plusieurs jeunes se disant pratiquants invoquaient des préceptes religieux. Ces discours ne sont pas propres à une religion particulière (chrétienne catholique, évangéliste, orthodoxe; musulmane) : c'est le niveau d'adhésion aux precepts et à la pratique religieuse qui jouent un rôle. Si la retenue ou sobriété sexuelle s'articule dans les discours des diverses tendances religieuses, les jeunes tendent à interpreter ces discours en termes de "tradition" transmise par les parents, renvoyant ainsi à ce qu'ils estiment être leur " africanité ».

\section{Préserver la sexualité féminine : un enjeu de la transmission intergénérationnelle}

Si les études conduites dans divers pays d'Afrique francophone montrent que les messages transmis dans l'éducation parentale en matière de sexualité sont de type conservateur, nous observons une continuité de ces messages venant des parents qui ont migré en Suisse. Certaines études montrent, par exemple, que le fait de tomber enceinte comporte le risque que l'enfant ne soit pas reconnu (Carle et Bonnet, 2009 ; Calvès, 2002 ; Mazzochetti, 2007), tout comme la perte de la virginité implique très souvent une baisse

\footnotetext{
${ }^{9}$ Comme le montrent les études sur les fils et filles d'immigrés en France (Hamel et al., 2011), et les recherches sur les jeunes Français d'origine maghrébine et les questions liées à la sexualité (Santelli, 2007 ; Clair, 2012).
} 
de valeur sur le marché matrimonial (Bledsoe et Cohen, 1993 ; Cole et Thomas, 2009 ; Mensch et al., 2006) ou le risque de ne pas trouver de mari (Attané, 2007 ; Hertrich, 2007). Ces messages sont particulièrement adressés aux jeunes femmes par les parents, la sexualité étant perçue comme une situation pouvant les fragiliser (perte de virginité, grossesse hors mariage) ou, à l'inverse, les sécuriser (mariage). Nous constatons, sur la base des assertions faites par nos interviewé.es, que ces messages se reproduisent dans la sphère familiale en contexte de migration. L'alerte pour éviter une grossesse "à tout prix " est une préoccupation parentale constante. Les parents, et notamment les mères, se dressent en gardiennes de la conduite et de la réputation des filles. Les messages contenus dans cette transmission intergénérationnelle se font sous forme d'alertes et leur contenu (éviter le rapport sexuel) est en décalage avec les recommandations transmises dans les cours d'éducation sexuelle qui visent, plutôt, à responsabiliser et autonomiser les jeunes en matière de choix sexuels, comme l'explique Bleue, une jeune femme congolaise de 21 ans, protestante et arrivée en Suisse il y a quatre ans. Elle a un permis de séjour stable et suit une formation professionnelle :

Tomber enceinte, à 16 ans, c'est pas la meilleure chose. Après dans tout ça, ça revient aux parents en fait. Si tu tombes enceinte ou bien que les parents apprennent que tu as eu des relations sexuelles, c'est quelque chose de très grave. Et puis les conséquences sont très lourdes. (Bleue)

Si le discours de Bleue se base sur les retombées sociales et notamment familiales dans le cas d'une transgression de la norme sexuelle (abstinence en dehors du mariage), Clara, jeune femme de deuxième génération, met en perspective ce décalage avec les normes transmises par les parents de ses amies suisses. De religion catholique, elle est née en Suisse et y vit depuis toujours. Le cap Vert, pays d'origine de ses parents, est pour elle une destination touristique exotique qu'elle a visitée quelques fois dans sa vie lors des vacances scolaires. Elle garde toutefois un rapport particulier avec la langue, le créole, qui la lie étroitement à ses parents et à leurs traditions. Pour elle, obéir ou laisser croire à ses parents qu'elle suit leurs normes en matière de sexualité relève d'une preuve de loyauté. La volonté d'éviter tout conflit ne traduit pourtant pas une adhésion totale aux valeurs des parents.

Quand je parle avec mes copines qui sont suisses, des fois je vois que c'est différent. Elles, depuis toutes jeunes, elles ont parlé de leur première fois, elles ont été chez le gynécologue avec leur maman, elles ont eu des moyens de contraception, et puis ça parle, ça parle ouvertement à la maison, autour d'un dîner, le soir, ou comme ça. Mais je vois que c'est différent, oui. C'est différent... Par exemple, avec mes parents c'est plus, comment dire, c'est plus tabou d'en parler, depuis toujours... Même là-bas, quand je vais en vacances, c'est des choses dont on ne parle pas avec les parents ou les grandsparents, on ne parle pas de ça, de ce thème. C'est dans la culture. On n'en parle pas. (Clara)

Les différences relevées par Clara et d'autres interviewé-es se justifient par le fait que leurs parents sont des immigrés. La comparaison entre " parents migrants " et " parents suisses " devient, dans les discours des jeunes, le seul argument pour expliquer la difficulté à parler de sexualité : les parents africains sont décrits comme "éduqués à l'ancienne », "vieux », porteurs d'une vision « traditionnelle » parce qu'ils sont migrants. 
Le décalage générationnel est ici pensé à l'aune de la migration et des origines, et non de manière transversale à d'autres générations de parents.

Lorsqu'on parle de sexualité, les discussions en famille sont décrites comme rares et ponctuelles, sous forme de rappels aux règles, de rappels à l'ordre ou d'alertes normatives comme l'expliquent les interviewées : « ne fais pas ceci » ou « fais attention à cela ».

Ben ils sont ouverts mais ils ne sont pas non plus... Ils ont quand même été éduqués un peu à l'ancienne. Si tu couches avec quelqu'un, c'est pour la vie. C'est un mec pour la vie. Ils n'ont pas la même pensée que dans les pays un peu plus ouverts, civilisés... Pas civilisés, mais plus ouverts où les gens se sentent plus libres de ce qu'ils veulent dire. Où les gens sont beaucoup moins... Ben, tu sors avec un garçon et c'est pour la vie. (Iris)

Iris, une Congolaise de 22 ans, est arrivée en Suisse à l'âge de 8 ans dans le cadre d'un regroupement familial. Elle travaille comme secrétaire et est bilingue (français et lingala). Pour elle, c'est la communication qui condense toutes les différences et constitue le signe manifeste de l'inadéquation à parler de sexualité. Les échanges sous forme de sommations ou d'avertissements sont la preuve pour elle d'un " manque de civilisation ». La démocratisation des libertés sexuelles (Fassin, 2006) traduit non seulement l'accès à la planification reproductive ou à l'avortement, mais aussi à une sexualité ludique, soit audelà et en dehors d'un projet de couple. Or, ces évolutions étant récentes et propres aux sociétés néolibérales (Weeks, 2014), les jeunes de deuxième génération se voient obligé es de trier ce qui peut être dit de ce qui doit être dissimulé aux parents. Plusieurs interviewées expliquent en détail les stratégies élaborées pour " sauver l'image " que les parents ont de leurs enfants, tout en cachant leur activité sexuelle.

Nos parents, ils nous ont dit: "pas de sexe avant le mariage ". Les moyens de contraception ils ne sont pas trop pour... Ils disent aussi : " quand on est sûr d'avoir trouvé la bonne personne, on va avoir des rapports et juste avec cette personne ». C'est un peu cette idée-là qu'ont nos parents et qu'ils nous répètent tout le temps... Les parents nous disent, on entend bien ce qu'ils nous disent [elle rit], mais pour nous c'est un peu dépassé ce qu'ils pensent. On les écoute, mais sans faire ce qu'ils nous disent... (Clara)

Comme le met en exergue Clara, d'autres jeunes ressentent ces obligations morales en termes de loyauté et de respect vis-à-vis des parents. Ceci est particulièrement vrai pour les femmes sur qui les attentes pèsent de manière plus explicite. Certaines décident alors d'adhérer volontairement au discours conservateur en matière sexuelle, en l'assumant pleinement. Dans ces cas, les jeunes se réapproprient les normes en les transformant en une nouvelle forme de maîtrise de soi : le renoncement et l'abstinence. Certaines femmes disent choisir l'abstinence sexuelle ou insistent sur une " sacralité » de la sexualité. Ces discours s'appuient souvent sur des préceptes religieux (toutes religions confondues) très souvent invoqués en entretien. Le message religieux est parfois utilisé comme contrenorme occidentale à une sexualité " trop libérée " qui deviendrait " débridée ", comme l'explique Tarah, 21 ans, née en Suisse d'une mère angolaise et d'un père congolais et poursuivant des études universitaires :

Je trouve que c'est important, je dirais que j'ai toujours trouvé important de ne pas avoir trop de partenaires, pour mon propre honneur, pour ma propre valeur, je trouve que c'est mieux de s'abstenir que de... On sait jamais si c'est la bonne personne, donc je 
préfère attendre de me marier et d'être sûre que c'est la bonne personne... Avant, je trouvais ça pas important, je croyais que c'était désuet, ça se faisait plus, mais après ben... J'ai avancé dans ma foi, c'est devenu quelque chose de plus important. Puis j'ai compris aussi mes propres valeurs à moi. Enfin, dans la société maintenant, je trouve que c'est plus important qu'une femme se préserve. (Tarah)

$\mathrm{Si}$ les attentes parentales, et a fortiori sociales, limitent les marges de manœuvre féminines, nous verrons dans la prochaine section comment - et cela est spécialement vrai pour les femmes - les jeunes s'appuient sur les expériences et confidences partagées entre pairs pour asseoir leur autonomie sexuelle (Bozon, 2012).

\section{Socialisation entre pairs et ajustement des rapports de genre}

Considérant la socialisation à la sexualité au sein des groupes de pairs au travers de leurs échanges, il émerge que, si les discussions sont genrées, un rapprochement de style et de contenu se produit entre hommes et femmes parmi les jeunes de deuxième génération. À l'instar d'études précédentes sur la sexualité des jeunes, notamment conduites en France (Blanchard et al., 2010), la tendance genrée vers une sexualité féminine plus responsable et romantique contre une sexualité masculine ludique et insouciante se nuance (Le Gall et Le Van, 2010). Ainsi, nous constatons que, dans notre population étudiée, l'écart entre hommes et femmes est moins important que celui entre les générations (entre migrant·es et fils ou filles de migrants). On observe les hommes de deuxième génération s'investir plus activement dans la contraception par exemple. Les femmes de deuxième génération, quant à elles, adoptent un langage qu'elles qualifient elles-mêmes de "vulgaire ", pour afficher leur agentivité sexuelle (Lang, 2011) qu'elle soit langagière ou mise en actes.

\section{Des responsabilités masculines et féminines : l'exemple de la contraception}

La sexualité est principalement discutée entre pairs et en particulier avec les ami-es du même sexe. L'amitié constitue un espace où les jeunes se sentent à l'aise pour s'exprimer et partager leurs expériences avec des personnes "qui comprennent ». Souvent les amitiés sont de longue date (fréquentation de la même école, résidence dans le même quartier). Les discussions, ainsi que les tons de l'échange, évoluent au fil du temps : le ton de la " rigolade » utilisé lors des premières discussions cède la place à celui des échanges plus sérieux. Bien que des hiérarchies existent à l'intérieur des groupes de pairs du même sexe - les plus expérimenté.es sont souvent les meneurs/meneuses des discussions -, la plupart du temps les conversations se basent sur l'échange des conseils pratiques qui fondent un savoir-faire sexuel et sentimental. La caractérisation en termes de genre concerne la manière d'aborder les sujets et les arguments plus que les thèmes euxmêmes. Les premières expériences sexuelles ou le choix du partenaire constituent des sujets de conversation féminine : les jeunes femmes mutualisent les récits des expériences des unes et des autres et en font une forme de savoir par expérience partagée. Le thème de la contraception illustre de manière idéaltypique les différences de genre quant aux manières de s'exprimer sur ce sujet par les femmes et les hommes. 
Les jeunes femmes affirment que la contraception est un sujet très discuté : le souci principal pour elles est d'éviter une grossesse, plus qu'une MST. Le risque de contracter le $\mathrm{VIH} /$ sida, ou d'autres MST, est perçu comme moins important que le risque de tomber enceinte suite à une relation sexuelle non protégée (Poglia Mileti et al., 2019). Cet aspect fait écho aux messages transmis par les mères et les alertes pour éviter à tout prix une grossesse. Leur mise en pratique au quotidien - à savoir l'adoption d'une contraception demande parfois des stratégies complexes, allant de la prise d'un contraceptif en cachette à l'élaboration de récits alambiqués pour organiser les sorties. Les femmes de deuxième génération sont plus sous pression, ayant souvent une interaction au quotidien avec leurs mères, à qui on attribue un rôle de surveillante des comportements sexuels à l'égard des autres membres de la communauté africaine en Suisse. Les femmes de première génération, quant à elles, se retrouvent plus souvent à gérer des relations inégalitaires et témoignent d'une sexualité féminine qui se construit de façon subordonnée à la sexualité masculine; notamment en ce qui concerne l'usage des contraceptifs et du préservatif (Poglia Mileti et al., 2019), comme le dit Diana, une Érythréenne de 21 ans, en Suisse depuis trois ans et insérée dans un cours d'apprentissage de langue française.

Quand on est amoureuse, on ne pense pas à ça [au risque de MST]... Je ne sais pas les autres mais pour moi, c'était comme ça... J'acceptais tout... (Diana)

Les jeunes hommes discutent aussi entre eux, mais s'ils partagent leurs expériences intimes, c'est avec un seul interlocuteur, souvent désigné comme le "meilleur ami ». Lorsque les discussions concernent la contraception ou la protection face aux risques sexuels, les hommes disent ne pas avoir besoin d'en discuter longuement. Pour eux, " une fois qu'on a reçu l'info, ça suffit ", ils la mettent en pratique et se débrouillent. Ils désignent les cours d'éducation sexuelle ou les campagnes de prévention comme les sources de leur savoir. L'importance de prouver une autonomie dans ce domaine est affirmée en entretien: la contraception ne constitue pas un thème privilégié de conversation entre hommes, il s'agit d'une affaire "à gérer " individuellement, comme l'explique Éric, 22 ans, né au Rwanda et arrivé en Suisse à l'âge de 5 ans :

Oui, toujours, en général, les filles veulent tout le temps se protéger. Moi, personnellement, je n'ai jamais essayé de ne pas me protéger. Moi-même, je ne peux pas ne pas me protéger et, enfin, ça va de soi en fait. C'est logique pour moi de me protéger et puis, pour la fille aussi. Donc, à moins qu'un des deux demande de ne pas se protéger, mais ça c'est jamais arrivé en tout cas pour moi. (Éric)

Dans la pratique, un écart se dessine entre hommes de première et de deuxième génération, ces derniers considèrent la protection comme quelque chose qui " va de soi », tandis que les premiers estiment que cela est une tâche plutôt féminine.

\section{Discussions entre pairs : la rigolade, les interdits et les conseils}

Les discussions masculines, lorsqu'elles ont lieu en groupe, " entre potes ", se déroulent sur le mode du rire ou de l'humour. Les thèmes peuvent varier, mais en général les questions tournent autour des nouvelles conquêtes amoureuses ou de l'importance d'informer les amis d'être sexuellement actif. Les appréciations ou les commentaires sur 
le corps des femmes se font publiquement et sont partagés en riant. Ces commentaires ont la fonction de montrer une capacité de séduction, plus que de prouver de véritables projets de conquête (Gourarier, 2017). Le langage jeune est souvent affiché, son usage diffère selon la maîtrise de la langue ou l'appartenance sociale (Sulstarova et al., 2019). À ce propos, les jeunes hommes de première génération semblent moins enclins à mobiliser des messages verbaux différents selon les interlocuteurs et les contextes d'interactions, dévoilant une moindre maîtrise des codes du langage sexuel, ayant été socialisés plus tardivement dans le contexte migratoire. L'extrait d'entretien avec Grégoire, 23 ans, né en Suisse de parents universitaires originaires du Ghana, et poursuivant ses études universitaires, illustre de manière représentative les activités de la « drague » masculine.

Là, toujours pas sérieux, jamais très sérieux. Quand on est entre garçons, c'est jamais la même chose que s'il y a une fille, même si elle n'a rien à voir. On va plus rester sur " tu as vu comme elle est belle sa coupe de cheveux " s'il y a une fille et, s'il n'y a pas la fille, on va dire " ah tu as vu ses fesses ? Tu as vu son corps? ». (Grégoire)

Les discussions sur la masturbation marquent un véritable décalage entre jeunes de première et de deuxième génération, et relèvent des représentations imprégnées de préceptes religieux. Les premiers considèrent ce sujet comme " tabou » et très honteux. II est presque impossible de le partager publiquement, y compris entre amis comme le dit Souma, un jeune Soudanais de 20 ans, en Suisse depuis trois ans.

Tu ne peux pas dire que tu fais ça avec ta main devant ton pote ! Ça ne se fait pas ! Même si tout le monde sait, on dit "j'ai une copine " même si tu n'en as pas. Je ne peux pas dire que je fais avec ma main tout le temps, ça ne se fait pas, sinon ils vont penser quoi de toi ? (Souma)

Grégoire, relancé sur le même sujet, se montre plus complice avec l'enquêtrice et admet que cela fait partie des manières de rigoler entre hommes et de se "chambrer ».

\begin{abstract}
Il me disait « vas-y masturbe toi, entretiens-toi »; là on se chambre: «là je te sens tendu... là ce serait le temps de te tirer une queue ». Ou bien... des choses... II dira par exemple " ouais, tu vois ta main ? Pense que ta main c'est ta meilleure amie pendant ces moments de solitude ». Disons si un pote vient et dit « aujourd'hui je me suis branlé deux fois", on va pas dire "mais de quoi tu parles ? c'est dégueulasse... ", on va presque plutôt dire " tape-là » et sûrement on va en rire. C'est pas un sujet tabou quoi. (Grégoire)
\end{abstract}

Quant aux discussions entre femmes de deuxième génération, on observe un changement évident dans la manière de parler et, le cas échéant, de valoriser l'activité sexuelle, dans le groupe d'amies. Si l'activité sexuelle doit être dissimulée ou diminuée visà-vis des parents et de la communauté, entre pairs, les discussions des jeunes femmes de deuxième génération sont similaires à celles des hommes, mettant en exergue le nombre de rapports sexuels, comme le dit Mila, une Congolaise de 20 ans, universitaire née en Suisse :

Elles en parlaient beaucoup [de leurs expériences]. Elles partageaient tout le temps, comme si c'était de l'exploit. [...] Elles le divulguaient parce que quand tu as de l'expérience, tu peux attirer encore plus de garçons. (Mila) 
Les espaces de sociabilité mixtes sont importants non seulement pour la drague mais

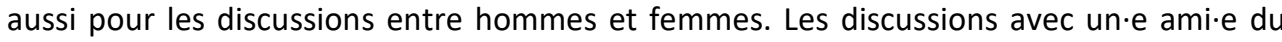
sexe opposé sont généralement considérées comme légères et se déroulent sur le registre de l'humour. L'ami-e du sexe opposé est considéré.e aussi comme une source d'informations et peut être perçu·e comme un.e représentant.e des goûts et des attentes du sexe opposé. L'intérêt de ces échanges est centré sur l'acquisition d'un bagage $d^{\prime}$ informations en matière de sexualité qui permet notamment de forger des techniques de drague. Kayla, une jeune Camerounaise de 19 ans, arrivée en Suisse à l'âge de 12 ans, l'explique ainsi :

En fait, les garçons ils donnent beaucoup plus de conseils. C'est ça quoi. Sans qu'on entre toutefois dans les détails comme avec mes copines, donc voilà. Ben tout ce qui touche à la sexualité, comment s'y prendre, comment se protéger, ce que les hommes aiment bien, voilà quoi. Avec les garçons, c'est des garçons, ils savent ce que les autres garçons aiment bien, du coup ils peuvent nous donner des conseils par rapport à ça. (Kayla)

Ceci est valable aussi pour les jeunes de première génération qui mettent en avant l'importance de parler avec quelqu'un du sexe opposé et idéalement plus expérimenté·e, comme l'explique David, un Sénégalais de 19 ans, en Suisse depuis un an et vivant dans un foyer pour demandeurs d'asile.

J'ai une amie qui est très proche, elle est mariée et a des enfants, elle a de l'expérience et des fois elle me guide un petit peu, ça aide, oui. Pour connaître les points faibles et les points forts. Ça aide. (David)

Finalement, les jeunes adaptent leur style selon le sexe de l'interlocuteur ou interlocutrice, tandis que l'origine n'est pas considérée. Les ami-es mentionné·es constituant le groupe de pairs ont par ailleurs des origines très variées (espagnole, italienne, albanaise, turque, etc.). En revanche, les jeunes de première génération disent faire parfois des "efforts " pour adapter leur style aux jeunes de deuxième génération (toutes origines confondues).

\section{Les libertés sexuelles aux prises avec les contraintes et les attentes familiales}

Les jeunes de première génération comparent leur contexte de socialisation avec le contexte de migration. Les différences sont relevées en termes de mœurs, d'habitudes, mais aussi de codes vestimentaires. Finalement tout les amène à souligner que dans le nouveau contexte il y a une "liberté majeure " : de l'accès à la contraception et au test de dépistage du VIH, à l'initiative des femmes et leurs libertés de fréquentation de l'espace public, aux codes vestimentaires sexualisant le corps des femmes. Si certain.es considèrent ces possibilités comme " une chance " et un pouvoir accru en termes de choix, d'autres soulignent les difficultés ou les contradictions entre ces modèles très permissifs et les contraintes sociales.

Les difficultés économiques, celles liées au statut juridique ou les problèmes liés aux documents ou permis de séjour tendent toutefois à restreindre la marge de manœuvre (fréquentation de pubs/clubs où vont les jeunes, déplacements, etc.) de certains jeunes et 
fragilisent les initiatives. En effet, pour les jeunes de première génération, les " libertés » découvertes en Suisse ne se traduisent pas forcément en opportunités réelles: les rencontres de partenaires potentiel-les demeurent difficiles et se déroulent dans un espace de séduction qui reste très compétitif. Pour d'autres, notamment les jeunes vivant en foyer, la question du lieu pour avoir un rapport sexuel demeure prioritaire.

Si les contraintes sont plus formelles et liées au pouvoir (d'action, d'achat), des attentes sociales sont aussi exprimées, en particulier vis-à-vis des femmes. Celles qui appartiennent à la deuxième génération, qui pourtant ont reçu des messages égalitaires dans le cadre scolaire, se voient renvoyées à leur position de filles de migrants, comme le dit Syra, 22 ans, d'origine angolaise, née en Suisse et étudiante universitaire, pour illustrer les enjeux du port d'un pantalon court :

Ma mère me dit " tu es africaine, tu ne vas pas te balader en mini short [...] il faut respecter ton corps ». Elle me dit ça, par exemple. Elle dit, par exemple, les shorts vous savez, les shorts de filles qui sont très courts où on voit presque les fesses - elle dit " ça c'est un truc de Blancs, c'est pas un truc que tu dois porter toi ». [...] Pour ma mère, $c^{\prime}$ est montrer son corps à tout le monde. Si tu portes des trucs courts, tu montres ton corps à tout le monde, ma mère elle dit " tu perds de la valeur ". Alors que si tu te caches et que tu montres juste ton corps à ton mari ou comme ça, c'est plus valorisant. (Syra)

$\mathrm{Si}$, de manière générale, les jeunes de deuxième génération, hommes et femmes, trouvent un contexte plus favorable en ce qui concerne l'accès aux services (planning familial, éducation sexuelle, accès aux contraceptifs, etc.) et aux droits sexuels en Suisse, ils ou elles sont soumis·es à un contrôle parental ressenti parfois comme plus important, ce d'autant plus qu'ils ou elles sont majoritaires à vivre encore avec leurs parents. Le risque qu'une relation sexuelle soit découverte par les parents est souligné à plusieurs reprises. Ce risque tient autant au fait de décevoir les attentes parentales qu'aux non-dits en matière sexuelle.

Encore une fois, l'écart entre les possibilités offertes et l'accès aux libertés sexuelles est plus grand pour les femmes qui se voient obligées d'élaborer des stratégies pour préserver leur réputation et cacher leur vie sexuelle, comme le dit Mélanie, 22 ans, étudiante universitaire, née en Suisse de parents congolais :

Les Africaines doivent faire les choses en cachette ! J'ai connu des filles qui disaient que par exemple c'étaient leurs parents qui leur avaient offert leurs premiers préservatifs ou bien... Ouais, enfin, que c'est eux qui les ont accompagnées pour le premier rendez-vous chez le gynécologue. Ça c'est des choses que moi j'ai toujours fait un peu en cachette et puis sur le fait accompli je suis obligée d'avouer ce que j'ai fait, quoi. C'est plus ça, ouais. J'aurais aimé pouvoir en parler plus ouvertement. (Mélanie)

Bien que les femmes de deuxième génération soient avantagées en matière de liberté sexuelle par rapport à celles de première génération, elles restent toutefois souvent prisonnières des stéréotypes inégalitaires opposant l'image de la " fille sérieuse " à celle de la "fille facile", opposition qu'elles estiment forte et répandue au sein de la communauté et auprès de la génération de leurs parents. 
La fameuse " réputation des filles » ça existe toujours ! Et justement, j'évite de coucher trop avec des gens qui se connaissent ou... de la région en fait ! (Mélissa)

Mélissa, 23 ans, née au Cameroun et arrivée en Suisse à l'âge de 6 ans, le dit clairement. Ce qui apparaît dans son discours, et celui d'autres femmes, c'est que ces restrictions ou ces inégalités existent parce que leurs parents sont "traditionnels" ou que les communautés africaines sont " conservatrices ». Le fait que les femmes se voient limiter leurs relations sexuelles, soient critiquées lorsqu'elles changent de partenaires ou adoptent un style vestimentaire trop voyant peut être interprété comme une forme de contrôle patriarcal de la sexualité féminine (Andro et al., 2010) et non pas par des facteurs culturels. Néanmoins, si les parents des amies suisses exercent des freins parfois similaires, les interviewées estiment que la vision de leurs parents est due à leurs origines (africaines), évoquant la nécessité de préserver une morale (sexuelle, esthétique) différente de celles des « Blancs ».

J'évite d'avoir plusieurs relations en une année... C'est-à-dire que ça fait un peu fille facile, en fait. Une femme qui ne sait pas ce qu'elle veut. Parce que nous on dit " peu importe le temps que ça va prendre, soit patiente ». Donc, il faut attendre. Et c'est un ou une dans la vie. C'est pour ça que les présentations dans les familles africaines c'est toujours compliqué... C'est ça, en fait. (Kayla)

L'origine des parents devient alors la raison pour laquelle les jeunes femmes doivent "faire gaffe » à ce qu'on dit d'elles, alors que ces inégalités existent dans d'autres communautés, notamment italiennes, espagnoles et latino-américaines (Carbajal, 2007). Par ailleurs, aucune étude à notre connaissance ne s'intéresse aux comportements et aux représentations des parents suisses en lien avec leur milieu de socialisation. Ainsi une " africanité " des inégalités réinvente et réaffirme à la fois les anciennes représentations raciales en décrivant les populations subsahariennes comme insensibles aux droits sexuels des femmes et justifiant ainsi des interventions sanitaires ciblées.

\section{Conclusions. Hiérarchies langagières et altérisation des sexualités en contexte de migration}

Les différences d'apprentissage, dans la gestion des discussions et des comportements, peuvent rendre les jeunes de première génération issu·es de la migration subsaharienne en Suisse moins préparé.es à se repérer dans un paysage où l'offre de services dans le domaine de la sexualité est riche mais demande pourtant à être sollicitée (offre médicale, offre scolaire, counseling au planning familial). S'adresser à un service est aussi le fruit d'une socialisation sexuelle que les jeunes né.es ou socialisées en Suisse apprennent à faire, ce qui n'est pas systématiquement le cas pour ceux et celles de première génération. La socialisation à un langage technico-médical dérivant de la santé sexuelle (Giami, 2015) et l'adhésion à un modèle médicalisant la sexualité produisent des hiérarchies dans la manière de se comporter mais aussi de penser les sexualités. La pluralisation des agents de socialisation due à l'expérience migratoire entraîne des changements dans le processus de transmission et d'intégration des références morales, sociales et culturelles en matière de sexualité. Cela place les jeunes - en particulier celles et ceux de la deuxième génération - dans des situations de conflits normatifs qui peuvent 
soulever des tensions intergénérationnelles, mettre à mal leurs sentiments d'allégeance ou au contraire renforcer la conscience de leur capacité à agir et à faire des choix.

Les jeunes femmes, dès leur socialisation en milieu scolaire mais aussi au sein de leur famille, tendent à être plus responsabilisées que les jeunes hommes vis-à-vis de leur corps et de leur sexualité. Les hommes de deuxième génération assument une charge contraceptive de manière plus volontaire que les jeunes migrants. Les femmes, quant à elles, se réapproprient un langage jusque-là réservé à la gent masculine ou valorisant l'activité sexuelle, ce qui renverse le modèle de la fille pudique. Cette dernière étant une norme transmise par l'éducation parentale, les femmes de deuxième génération tendent très souvent à dissimuler leurs relations sexuelles vis-à-vis des parents et membres des communautés africaines. Le risque d'être considérée comme une " fille facile » entame à la fois la réputation et l'image que les parents africains en Suisse veulent préserver de leurs filles. L'enjeu symbolique de la transmission intergénérationnelle d'une morale sexuelle " africaine " touche plusieurs aspects : éviter le risque de grossesse, trouver le "bon partenaire", adopter un code vestimentaire respectable, etc. Une forme de concurrence et de hiérarchie culturelle et raciale se joue ainsi sur le terrain de l'éducation sexuelle. Pour les jeunes issu·es de la migration africaine en Suisse, les parents, restés en Afrique ou ayant migré en Suisse, représentent le passé et une vision opposée aux valeurs circulant entre pairs. La tradition est évoquée et dépeinte comme allant à l'encontre d'une égalité entre hommes et femmes dans la sexualité. Les jeunes considèrent que cette égalité va de soi en Suisse, alors que cela relève d'une idéalisation de l'autre et d'une altérisation des normes sexuelles parentales.

\section{Bibliographie}

Amsellem-Mainguy Y., Vuattoux A., 2018, Enquêter sur la jeunesse. Outils, pratiques d'enquête, analyses, Paris, Armand Colin.

Andro A., Bachmann L., Bajos N., Hamel C., 2010, "La sexualité des femmes : le plaisir contraint », Nouvelles Questions Féministes, vol. 29(3), pp. 4-13.

Attané A., 2007, "Choix matrimoniaux : le poids des générations. L'exemple du Burkina Faso ", in P. Antoine (éd.), Les relations intergénérationnelles en Afrique : approche plurielle, Nogent-surMarne, Éditions CEPED, pp. 167-195.

Barrense-Dias Y., Akre C., Berchtold A., Leeners B., Morselli D., Suris J-C., 2018, Sexual health and behavior of young people in Switzerland, Lausanne, Institut universitaire de médecine sociale et préventive, Raisons de santé, $n^{\circ} 291$.

Blanchard V., Revenin R., Yvorel J., 2010, Les jeunes et la sexualité : Initiations, interdits, identités $\left(X I X^{e}-X X I^{e}\right.$ siècle), Paris, Éditions Autrement.

Bledsoe C.H., Cohen B., 1993, Social Dynamics of Adolescent Fertility in Sub-Saharan African, Washington D.C., National Academy Press.

Bolzman C., Gakuba T.-O., Guisé I., 2011, Migrations des jeunes d'Afrique subsaharienne : quels défis pour l'avenir ?, Paris, L'Harmattan.

Bozon M., 2013, Sociologie de la sexualité ( $3^{e}$ édition.), Paris, Armand Colin. 
Bozon M., 2012, "Autonomie sexuelle des jeunes et panique morale des adultes : Le garçon sans frein et la fille responsable ", Agora débats/jeunesses, n 60(1), pp. 121-134.

Bozon M., 1999, "Les significations sociales des actes sexuels ", Actes de la recherche en sciences sociales, $\mathrm{n}^{\circ} 128$, pp. 3-23.

Campisi L., Berchtold A., Barrense-Dias Y., Akré C., Gehri M., Surís J.-C., 2017, « Risk behaviours among native and immigrant youths in Switzerland: a cross-sectional study ", Swiss Medical Weekly, ${ }^{\circ} 147$, w14531.

Carle J., Bonnet D., 2009, " Identité et question des origines dans l'abandon d'enfants au Burkina Faso ", Anthropologie et sociétés, n³3(1), pp. 141-155.

Calvès A.E. 2002, "Abortion risk and abortion decision making among youth in urban Cameroon ", Studies in Family Planning, ${ }^{\circ} 33(3)$, pp. 249-260.

Carbajal M., 2007, "Femmes latino-américaines sans-papiers en Suisse. Analyse de projets migratoires, logiques d'action et modes de vie ", in C. Gavray (éd.), Femmes et mobilités, Marcinelle, Éditions Cortext, pp. 367-385.

Clair I., 2012, "Dedans/dehors. La sexualité, une ligne de démarcation ? », Genre, sexualité \& société, $\mathrm{n}^{\circ} 7, \mathrm{DOI}: 10.4000 / \mathrm{gss} .2386$.

Cole J., Thomas L.M., 2009, Love in Africa, Chicago, University of Chicago Press.

Colombo A., Carbajal M., Carvalhosa Barbosa M., Tadorian M. (2017), « Gagner la reconnaissance des pairs en évitant la réputation de "pute". L'injonction paradoxale qui pèse sur les filles impliquées dans les transactions sexuelles ", Revue Jeunes et Société, n² 2(2), pp. 70-93.

Desgrées du Loû A., Lert F., 2017, Parcours. Parcours de vie et de santé des Africains immigrés en France, Paris, La Découverte.

Efionayi-Mäder D., Ruedin D., 2017, État des lieux du racisme anti-Noir-e en Suisse. Étude exploratoire à l'attention du Service de lutte contre le racisme (SLR), Neuchâtel, SFM Studies \#67f.

Efionayi-Mäder D., Pecoraro M., Steiner I., 2011, La population subsaharienne en Suisse : un aperçu démographique et socio-professionnel, Neuchâtel, Études du SFM 57.

Efionayi-Mäder D., Moret J., Pecoraro M., 2005, Trajectoires d'asile africaines. Déterminants des migrations d'Afrique occidentale vers la Suisse, Neuchâtel, Études du SFM 38A.

Elias N., 1973, La civilisation des mœurs, Paris, Calmann Lévy.

Fassin É., 2006, "La démocratie sexuelle et le conflit des civilisations », Multitudes, n²6(3), pp. 123-131.

Gardey D., Vuille M., 2018, Les sciences du désir. La sexualité féminine, de la psychanalyse aux neurosciences, Lormont, Éditions Le Bord de l'Eau.

Giami A., 2015, "Sexualité, santé et droits de l'Homme : l'invention des droits sexuels », Sexologies Revue européenne de sexologie et de santé sexuelle, $\mathrm{n}^{\circ}$ 24(3), pp. 105-113.

Giddens A., 1992, The Transformation of Intimacy: Sexuality, Love and Eroticism in Modern Societies, Stanford, Stanford University Press.

Gourarier M., 2017, Alpha mâle. Séduire les femmes pour s'apprécier entre hommes, Paris, Éditions Le Seuil. 
Hamel C., Moguérou L., Santelli E., 2011, "L'entrée dans la vie adulte des filles et fils d'immigrés », Politiques sociales et familiales, $n^{\circ} 105$, pp. 47-58.

Hertrich V., 2007, « Nuptialité et rapports de genre en Afrique. Tendance de l'entrée en union, 195099 », in T. Locoh (Éd.), Genre et société en Afrique, Paris, Édition de l'INED, pp. 281-307.

Lang M., 2011, "L'“agentivité sexuelle' des adolescentes et des jeunes femmes : une définition ", Recherches féministes, $\mathrm{n}^{\circ} 24(2), \mathrm{pp} .189-209$.

Le Gall D., Le Van C., 2010, "Le premier rapport sexuel : récits féminins versus récits masculins », Agora débats/jeunesses, $\mathrm{n}^{\circ}$ 56(3), pp. 63-72.

Marquet J., 2004, Normes et conduites sexuelles. Approches sociologiques et ouvertures pluridisciplinaires, Louvain-la-Neuve, Éditions Academia.

Mazzocchetti J., 2007, « De l'autorité à l'affect : transformation des paternités au sein de la jeunesse ouagalaise scolarisée (Burkina Faso) ", Recherches sociologiques et anthropologiques, $n^{\circ} 38(2)$, pp. 47-64.

Mensch B., Grant M.J., Blanc A., 2006, "The Changing context of sexual initiation in sub-Saharan Africa», Population Development Review, n 32(4), pp. 699-727.

Poglia Mileti F., Mellini L., Sulstarova B., Villani M., Singy P., 2019, «Exploring barriers to consistent condom use among sub-Saharan African young immigrants in Switzerland ", AIDS Care, $n^{\circ} 31(1)$, pp. 113-116.

Santelli E., 2007, Grandir en banlieue. Parcours et devenir de jeunes Français d'origine maghrébine, Paris, Éditions CIEMI.

Sulstarova B., Poglia Mileti F., Mellini L., Villani M., Singy P., 2019, « Parler de sexualité : le point de vue des jeunes migrant.e.s subsaharien.ne.s", Actes du colloque de la Société internationale de linguistique fonctionnelle, Moscow City University, pp. 121-124.

Villani M., Poglia Mileti F., Mellini L., Sulstarova B., Singy P., 2014, " Les émotions au travail (scientifique): enjeux éthiques et stratégies méthodologiques d'une enquête en terrain intime ", Genre, Sexualité \& Société, n 12, DOI : 10.4000/gss.3333.

Villani M., Poglia Mileti F., Mellini L., Sulstarova B., Singy P., 2015, «L'engagement (scientifique) sensible. Stratégies d'enquête sur les thèmes de la sexualité, la séropositivité et le secret ", Civilisations, $n^{\circ}$ 64(1), pp. 43-54.

Wandeler G., Dubois-Arber F., Clerc O., Cavassini M., 2008, "Épidémiologie du VIH », Revue médicale suisse, $n^{\circ} 4$, pp. 888-93.

Weeks J., 2014, Sex Politics and Society. The Regulation of Sexuality since 1800, New York, Routledge. 